\title{
GUIDELINES
}

\section{Organização de Serviços de Apoio Clínico para Doentes com Cefaleias em Portugal}

\section{Headache Service Organization and Delivery in Portugal}

\author{
PROPOSTA DA SOCIEDADE PORTUGUESA DE CEFALEIAS
}

\author{
Daquel Gil-Gouveia ${ }^{1,2, \star}$, Liliana Pereira ${ }^{1,3}$, (D) Sara Machado 1,4, (D) Elsa Parreira 1,2,4 \\ 1-Direção da Sociedade Portuguesa de Cefaleias; \\ 2-Centro de Cefaleias, Serviço Neurologia, Hospital da Luz, Lisboa, Portugal; \\ 3-Serviço de Neurologia, Hospital Garcia d'Orta, Almada, Portugal; \\ 4-Serviço de Neurologia, Hospital Fernando Fonseca, Amadora, Portugal.
}

A Sociedade Portuguesa de Cefaleias, pretende com este documento melhorar a eficiência e qualidade da prestação de serviços médicos aos doentes com cefaleias em Portugal.

The Portuguese Headache Society, intends, with this document, to improve the efficiency and quality of the provision of medical services to headache patients in Portugal.

DOI: https://doi.org/10.46531/sinapse/GU/200034/2021

Informações/Informations: Guidelines, publicado em Sinapse, Volume 21, Número 2, abril-junho 2021. Versão eletrónica em www.sinapse.pt Guidelines, published in

Sinapse, Volume 21, Number 2, April-June 2021. Electronic version in www.sinapse.pt (c) Autor (es) (ou seu (s) empregador (es)) e Sinapse 2021. Reutilização permitida de acordo com CC BY-NC. Nenhuma reutilização comercial. (C) Author(s) (or their employer(s)) and Sinapse 2021. Re-use permitted under CC BYNC. No commercial re-use.

\section{Palavras-chave:}

Cefaleias;

Cuidados de Saúde Primários:

Neurologia;

Normas de Orientação.

Keywords:

Headache;

Guidelines;

Neurology;

Primary Health Care.

\section{*Autor Correspondente Corresponding Author: Raquel Gil-Gouveia Hospital da Luz Lisboa Avenida Lusíada 100 1500-650 Lisboa, Portugal rgilgouveia@gmail.com}

Recebido / Received: 2020-07-08 Aceite / Accepted: 2020-08-27 Publicado / Published: 2021-07-29

\section{Resumo}

As cefaleias são patologias com de elevada prevalência populacional e de impacto substancial em termos de saúde pública, sendo atualmente a segunda causa mundial de anos vividos com incapacidade. $\mathrm{O}$ acesso destes doentes ao tratamento certo, no momento certo tem o potencial de diminuir substancialmente o impacto individual, social e económico destas patologias.

Neste sentido, a Sociedade Portuguesa de Cefaleias, seguindo as orientações da European Headache Federation e da campanha Lifting-the-Burden (com relações oficiais com a Organização Mundial de Saúde), elaborou a presente proposta de definição da organização dos serviços de apoio clínico para doentes com cefaleias em Portugal.

Neste documento pretende-se adaptar à realidade portuguesa as recomendações internacionais, avaliando a da necessidade de cuidados em cefaleias em Portugal e organizando o consumo de recursos de saúde através de um modelo de integração de cuidados multinível - desde a educação para a saúde, atendimento nos cuidados de saúde primários e referenciação para consultas de neurologia ou monotemáticas em cefaleias e/ou centros de cefaleias ou unidades de referência.

\footnotetext{
Abstract

Headaches are very prevalent disorders with substantial impact in terms of public health, being currently the second worldwide cause of years lived with disability. Headache patients' access to the right treatment at the right time has the potential to substantially decrease the individual, social and economic impact of these disorders.

With this purpose, the Portuguese Headache Society, following the guidelines of the European Headache Federation and the Lifting-the-Burden campaign (with official relations with the World Health Organization), prepared a proposal to define the organization of clinical support services for headache patients in Portugal.

The present document intends to adapt the international recommendations to the Portuguese reality, assessing the need for headache care in Portugal and organizing health resources through integrated multilevel care model - from health education, primary health care and referral for neurology or monothematic consultations in headache and / or headache centers or specialized units.
} 


\section{Introdução}

As cefaleias representam um grupo de patologias extremamente frequentes e diversas, cujo impacto oscila entre situações raras, autolimitadas e de baixo impacto (por exemplo a cefaleia por compressão externa) a situações de risco vital e emergentes (por exemplo a cefaleia súbita explosiva).' As patologias mais frequentes são as cefaleias primárias, cujo impacto é de enorme relevância em termos individuais (de sofrimento, incapacidade temporária recorrente e perda de qualidade de vida) e ainda maior em termos de saúde pública. Destas, o paradigma é a enxaqueca, atualmente considerada a primeira causa de anos vividos com incapacidade nos indivíduos com menos de 50 anos $^{2}$ e a mais relevante em termos de impacto económico, sobretudo por perda de produtividade laboral. ${ }^{3-5}$ Este impacto é mitigável, dado que o diagnóstico destas patologias é acessível e de baixo custo e existem várias opções terapêuticas válidas e eficazes. Apesar disto, há evidência de subdiagnóstico e que as terapêuticas não são disponibilizadas de forma eficiente e equitativa. ${ }^{6,7}$ Por outro lado, o grupo das cefaleias secundárias, embora menos expressivo em termos de frequência e de impacto populacional (dado que as cefaleias são, neste grupo, habitualmente um dos sintomas de outra patologia) tem enorme impacto individual e relevância clínica, dado que podem colocar problemas de diagnóstico diferencial e orientação diagnóstica e terapêutica que são difíceis de ultrapassar se os clínicos tiverem pouca formação nestas patologias.

Pelo exposto, a Sociedade Portuguesa de Cefaleias, entidade científica que a nível nacional se dedica ao estudo das cefaleias e ao desenvolvimento da formação pós-graduada em cefaleias, reunindo os profissionais com diferenciação específica nesta área, em sintonia com a European Headache Federation e a campanha Lifting-the-Burden (com relações oficiais com a Organização Mundial de Saúde) considera que é de extrema relevância uma proposta de definição da organização dos serviços de apoio clínico para doentes com cefaleias em Portugal. $\mathrm{O}$ objetivo ultimo é garantir a qualidade destes serviços, ou seja, que os serviços corretos estejam disponíveis a quem deles necessita de forma atempada, proporcionada, eficiente e custo-efetiva. ${ }^{8}$

Neste documento foi efetuada a transposição e adequação para a realidade nacional das recomendações europeias a respeito da organização dos serviços de saúde para os doentes com cefaleias ${ }^{8-10}$ assim como da qualificação necessária dos profissionais " envolvidos nestas unidades. Não se pretende, neste documento, determinar as necessidades formativas pré ou pós-graduadas dos profissionais de saúde envolvidos, assim como não são objetivos estabelecer critérios de diagnóstico, indicações para a investigação complementar ou orientações terapêuticas - todos estes aspetos são avaliados independentemente, em documentação e sede própria. No entanto, dado que a qualificação profissional está intrinsecamente ligados com as recomendações de organização de serviços, presume-se que será influenciada e influenciará as mesmas, pelo propósito final de meIhorar a qualidade vida daqueles que são afetados por cefaleias. $^{12}$

\section{Avaliação da necessidade de cuidados em cefaleias em Portugal}

Estudos populacionais estimam que, por cada I 000000 de pessoas que vivam na Europa, existam?:

- I 10000 adultos com enxaqueca, dos quais 90000 com incapacidade significativa;

- 600000 adultos com cefaleias tipo tensão não necessariamente incapacitantes;

- 30000 adultos com cefaleias diárias ou quase diárias, incapacitantes e cuja maioria utiliza analgésicos de forma excessiva.

Daqui se depreende que cerca de 120000 adultos por cada I milhão de habitantes (aproximadamente $12 \%$ da população) teria benefício no tratamento das suas cefaleias, o que é suportado por dois estudos, um no Reino Unido onde cerca de 17\% das consultas dos médicos de Medicina Geral e Familiar (MGF) são por cefaleias, e outro na Dinamarca, revelando que II\% da população foi ao médico por cefaleias, no último ano. ${ }^{9}$

Considerando os dados portugueses, ${ }^{13}$ que revelam uma prevalência de enxaqueca episódica de 19,97\% (dos quais $37 \%$ com mais de 4 dias por mês com dor), $23,5 \%$ de cefaleias crónicas (dos quais I,5\% com enxaqueca) e $70,5 \%$ de cefaleias tipo tensão (dos quais $65 \%$ episódicas) e a população recenseada em $2011,{ }^{14}$ as estimativas seriam:

- 2100167 (ou 200000 por milhão) de adultos com enxaqueca episódica, dos quais 780392 (ou 74000 por milhão) com incapacidade significativa (e provável indicação para profilaxia);

- 6859 II5 (ou 650000 por milhão) com cefaleias tipo tensão não incapacitantes;

- 747620 (ou 7I 000 por milhão) com cefaleias crónicas (enxaqueca e cefaleia tipo tensão) incapacitantes.

Por analogia, estimar-se-ia que cerca de 145000 adultos por cada I milhão de habitantes (aproximadamente $14,5 \%$ da população) terá benefício no tratamento das suas cefaleias em contexto de cuidados de 
saúde. Assume-se que apenas $50 \%$ dos indivíduos que beneficiariam de cuidados de saúde em relação às suas cefaleias efetivamente procurem esses cuidados de saúde, ${ }^{9}$ que são quase universalmente prestados em âmbito de cuidados ambulatórios; a estimativa de internamento nesta patologia é inferior a $1 \% .{ }^{9}$ Considerando que a organização do Serviço Nacional de Saúde Português é efetuada por níveis de proximidade e diferenciação técnica, propõe-se que a gestão destas patologias respeite a mesma abordagem e o plano de redes de referenciação, ${ }^{15}$ de acordo com o exposto de seguida.

Estima-se que $90 \%$ destes doentes apresentem quadros clínicos pouco complexos que, embora necessitem de acompanhamento médico, não requerem conhecimento técnico-científico diferenciado e podem/devem idealmente ser geridos nas unidades funcionais de cuidados de saúde primários, ou seja, cerca de 65250 indivíduos por milhão vão requerer avaliação por médicos especialistas em MGF, devido a queixas de cefaleias.

Cerca de $10 \%$ dos doentes serão casos de maior dificuldade de gestão clínica, devido quer à complexidade e agressividade da patologia, mas frequentemente também pela existência de comorbilidades que limitam ou dificultam a eficácia das estratégias disponíveis. Estes tem indicação para serem avaliados em cuidados mais diferenciados que são, no contexto Português, consultas de Neurologia, existentes em centros de nível II/ III/ IV. ${ }^{16}$ Assim, 7250 indivíduos por milhão necessitam de avaliação e seguimento em consulta de Neurologia, devido a queixas de cefaleias.

Destes, cerca de 10\% (1\% do total, ou seja I 450 por milhão - 14500 a nível nacional) terão cefaleias complexas com comorbilidades múltiplas e necessitarão de abordagem multidisciplinar, dedicada, com técnicas e terapêuticas diferenciadas e por vezes necessidade de internamento, pelo que deverão ser avaliados em sede de consulta dedicada, idealmente multidisciplinar e associada a centro de investigação/formação ou académico na área das cefaleias - centros de nível I. ${ }^{16}$ Prevendo-se nos próximos anos a emergência de marcada inovação terapêutica nesta área, poderá ser inclusivamente proposta a criação de Centros de Referência em Cefaleias, à semelhança dos existentes para outras patologias, ${ }^{17}$ reconhecendo a necessidade de especialização das alternativas terapêuticas, acompanhada de avaliação dos cuidados e dos resultados, assim como com a implementação sistemática da investigação e formação. Estes tipos de centros necessitam de enquadramento no modelo atual de referenciação, financiamento e de contratualização. ${ }^{16}$
A taxa de procura de terapêutica deste conjunto de indivíduos severamente afetados será potencialmente superior a 50\%, embora não se conheçam estimativas.

Todas as estimativas apresentadas não implicam que os restantes doentes com cefaleias (a maioria, 776000 por milhão) não tenham indicação para assistência médica devido às suas cefaleias, mas que provavelmente terão cefaleias que são geríveis quer com automedicação, quer com orientações simples e pontuais, admitindo-se que o consumo de recursos de serviços de saúde destes indivíduos seja pouco representativo.

Por outro lado, estes valores justificam a necessidade imperativa de incluir as cefaleias numa política construtiva e racional de educação para a saúde da população, nomeadamente em relação a sinais de alarme das cefaleias, estilos de vida saudáveis e adoção de medidas não farmacológicas, indicações para seguimento médico e estratégias eficientes e riscos da automedicação e sobre a estrutura de apoio médico instalada para tratar as cefaleias. Propõe-se, desta forma, a inclusão da temática das Cefaleias no Programa Nacional de Educação para a Saúde, Literacia e Autocuidados. ${ }^{18}$

A Sociedade Portuguesa de Cefaleias tem, como um dos seus objetivos primordiais, a divulgação de conhecimentos científicos sobre cefaleias e está preparada para colaborar a todos os níveis - quer na prestação direta de esclarecimentos à população, colaboração com associações de doentes e colaboração com a DGS e cuidados de saúde primários, assim como no ensino médico pré-graduado e pós-graduado, onde tem centrado a sua atividade nos últimos anos. ${ }^{19}$

\section{Consumo de Recursos a cada nível de prestação de cuidados}

\section{I Cuidados de saúde primários}

Pressupõe-se neste tópico que os médicos especialistas em MGF tenham formação e treino em cefaleias durante o seu internato médico. Embora não esteja definido nenhum estágio obrigatório neste tópico, ${ }^{20}$ o tema será certamente abordado na rotina clínica do dia-a-dia, dado o número elevado de consultas devido a cefaleias, cerca de 4,4/100 doentes/ano (6,4 nas mulheres e 2,5 nos homens). ${ }^{21}$ A existência de um estágio opcional em Neurologia permite, aos médicos internos que o desejem, ter maior proximidade e conhecimento mais diferenciado nesta patologia. Por outro lado, a Sociedade Portuguesa de MGF tem oferecido formação em Neurologia (incluindo em cefaleias) nas suas escolas, assim como a SPC tem divulgado os seus cursos a internos de MGF, no sentido de otimizar o conhecimento médico 
dos especialistas em MGF sobre o diagnóstico, abordagem e tratamento das cefaleias mais comuns. Será desejável, dada a prevalência destas patologias - superior à prevalência da diabetes e asma, ${ }^{22}$ por exemplo - que o treino dos especialistas em MGF incluísse obrigatoriamente formação em cefaleias.

A realidade nacional sobre a perceção, prevalência e gestão da enxaqueca dos médicos (especialistas em MGF e Neurologia), embora retratada de forma não generalizável em 2009, revelou que a maioria dos profissionais tem perceções adequadas e realistas sobre a doença e utiliza medicação recomendada nas orientações terapêuticas da Sociedade Portuguesa de Cefaleias. ${ }^{23}$

As recomendações internacionais referem que, a este nível, serão essenciais as seguintes competências":

- ter, pelo menos, completado um curso teórico sobre cefaleias;

- ter adquirido as habilitações e competências de forma a ser capaz de efetuar o diagnóstico e gerir a maioria dos doentes com enxaqueca episódica e crónica (com e sem aura) e com cefaleia tipo tensão episódica, de acordo com as orientações diagnósticas e terapêuticas nacionais ${ }^{24,25}$ e/ou europeias $^{26,27}$;

- reconhecer adicionalmente outras cefaleias primárias (cefaleia tipo tensão crónica, cefaleia em salvas episódica ou crónica) e secundárias consideradas essenciais (cefaleia pós-traumática, cefaleia atribuída a hemorragia subaracnoideia, a arterite temporal, a aumento da pressão intracraniana, por uso excessivo de medicação analgésica, por infeção intracraniana, por hipertensão arterial, por glaucoma agudo) e nevralgia do trigémio clássica ou idiopática;

- manter as suas habilitações através da prática clínica rotineira, dedicando o equivalente a meio-dia de consultas ( 3,5 horas) por semana a doentes com cefaleias, em média;

- manter-se atualizado no tema através da participação em, pelo menos, um momento formativo em cefaleias, com uma duração mínima de 4 horas, a cada 3 anos.

Aos especialistas em MGF é também solicitada a função de referenciação para cuidados mais diferenciados, cabendo-lhes identificar os $10 \%$ dos indivíduos com benefício em ser acompanhados em contexto de consulta de neurologia, ou mesmo os $1 \%$ de doentes que terão indicação para ser orientados para centro terciário/consulta multidisciplinar ou centro académico dedicado às cefaleias.

Os médicos especialistas em MGF tem ainda um pa- pel extremamente relevante, dada a sua proximidade às comunidades, na Educação e Literacia para a Saúde, a ser efetuada em contexto individual, familiar e, idealmente, comunitário. Propõem-se assim a realização de sessões de esclarecimento, a distribuição de folhetos informativos e outras iniciativas enquadráveis neste âmbito.

Assume-se, adaptando as recomendações internacionais ${ }^{10}$ e de forma a ser possível estimar o tempo de trabalho médico necessário para gerir estes doentes, que um adulto com cefaleias gerido nos cuidados de saúde primários consumirá 1 hora de tempo médico a cada 2 anos ( 3 consultas de 20 minutos), admitindo-se por motivos pragmáticos que não existirão faltas às consultas.

Desta forma, por cada 100000 utentes, 6525 solicitarão consulta de MGF por cefaleias, que representam 19575 consultas a cada 2 anos, ou seja, 9788 por ano ou 204 por semana útil. Estes valores representam um investimento temporal de 3262 horas por ano, ou seja, 68 horas por semana ou o equivalente a dois médicos de MGF em horário completo apenas dedicados às cefaleias, por cada 100000 utentes. Extrapolando para a população portuguesa, necessitaríamos do equivalente a 200 médicos de MGF a trabalhar a tempo inteiro em cefaleias em Portugal.

Admitindo o rácio indicativo de 1500 utentes por profissional especialista em $\mathrm{MGF}^{28}$ cada médico teria 98 utentes que necessitariam de acompanhamento por cefaleias, gerando 147 consultas por ano, ou aproximadamente I hora/semana, que é, em média, insuficiente para manter a prática clínica. Estratégias para colmatar esta limitação podem passar por aumentar o tempo de formação ou efetuar breves estágios de requalificação em centros especializados. Uma alternativa seria, à semelhança da estrutura existente no Reino Unido, existir por unidade de saúde familiar, um colega de MGF que se dedique às cefaleias e que tenha mais tempo dedicado para esta patologia.

\subsection{Consultas de Neurologia ou monotemáticas em cefaleias}

Os médicos especialistas em neurologia têm formação e treino em cefaleias durante o internato médico, muito embora não esteja definido nenhum estágio obrigatório neste tópico ${ }^{29}$; o tema, dada a sua prevalência, é um dos grandes temas da Neurologia Clínica e é objeto obrigatório de treino e de avaliação num candidato a especialista em Neurologia. A International Headache Society propõe um currículo mínimo de treino em cefaleias para neurologistas," que inclui aspetos sobre a 
anatomia, fisiopatologia, epidemiologia, ambiente, incapacidade, genética, investigação clínica das cefaleias, diagnóstico, classificação, terapêutica, curso e prognóstico dos vários tipos de cefaleias, assim como das suas comorbilidades mais frequentes. Os especialistas em Neurologia têm oportunidade de atualização frequente de conhecimentos, existindo programas de atualização europeus/internacionais (cursos e congressos da International Headache Soiety (IHS), European Headache Federation (EHF), Migraine Trust e European Academy of Neurology (EAN)), escolas de verão da EHF e congressos e cursos nacionais, promovidos pela SPC e SPN. Para quem deseje efetuar formação avançada, existem dois programas europeus de Mestrado ${ }^{30,31}$ em cefaleias, sendo adicionalmente possível efetuar doutoramento na área em qualquer universidade que ministre esta especialização no campo das Neurociências.

Recomenda-se, com base nas orientações internacionais que, a este nível, serão essenciais as seguintes competências 9 :

- ter adquirido as habilitações e competências de forma a ser capaz de efetuar o diagnóstico e gerir a maior parte dos doentes com cefaleias primárias, de acordo com as orientações diagnósticas e terapêuticas nacionais ${ }^{24,25}$ e/ou europeias, ${ }^{26,27}$ assim como a maior parte das cefaleias secundárias;

- manter as suas habilitações através da prática clínica rotineira, dedicando o equivalente a um terço do seu tempo de consulta semanal a doentes com cefaleias, em média;

- manter-se atualizado no tema, através da participação em pelo menos um momento formativo em cefaleias, com uma duração mínima de 6 horas, a cada 2 anos;

- manter a comunicação com os colegas de MGF, quer recebendo, quer retornando os casos que obtenham uma solução estável com um plano delineado e não necessitem manter seguimento em consulta de Neurologia.

No tocante à estimativa do tempo de trabalho médico necessário a este nível, ${ }^{10}$ admite-se que um adulto com cefaleias, gerido na consulta de Neurologia consumirá 2 horas e 30 minutos de tempo médico a cada 2 anos $(3+2$ consultas de 30 minutos, o tempo mínimo recomendado para uma consulta de Neurologia). ${ }^{32}$

Por cada 100000 utentes, 725 necessitarão de consulta de Neurologia por cefaleias, representando 3625 consultas a cada 2 anos, ou seja, 1812 por ano ou 38 por semana útil. Estes valores representam um investimento temporal de 906 horas por ano, ou seja, 17 horas por semana ou o equivalente a $42,5 \%$ do tempo de um médico neurologista em horário completo (40 horas semanais) apena dedicado às cefaleias, por cada I00 000 utentes (I por 200 000). Extrapolando para a população portuguesa, necessitaríamos do equivalente a 50 médicos neurologistas a trabalhar a tempo inteiro em cefaleias, em Portugal.

Considerando a estrutura da rede de referenciação hospitalar, ${ }^{16}$ sugere-se que nos centros de nível III/IV estas consultas sejam integradas na consulta de neurologia, dado que cobrem uma população de 150 a 300000 habitantes e dispõem de uma média de 4 a 7 profissionais, conforme a dimensão da área de referência. ${ }^{33}$ Nestes centros, por cada 200000 habitantes há necessidade de um neurologista a tempo inteiro, pelo que se sugere a distribuição destes doentes por 3 a 4 especialistas, garantindo a continuidade de cuidados e um volume adequado para manter a experiência clínica.

Nos centros de nível II, ${ }^{16}$ em que a população de referência são 300000 a I milhão de habitantes e existem 12 ou mais especialistas, ${ }^{33}$ serão necessários 2 a 5 especialistas com dedicação completa às cefaleias, conforme a sua dimensão. Nestas unidades, o volume de trabalho (pelo menos 40 horas semanais de consulta por cada 200000 utentes) poderá justificar a criação de uma consulta dedicada às cefaleias, garantida por, pelo menos, 3 a 4 profissionais em tempo parcial (cada um com pelo menos 2 turnos de 5 a 6 horas por semana), por cada 300000 utentes. Nestas consultas monotemáticas podem ser implementadas algumas medidas de apoio que auxiliam em termos de gestão, como a implementação de secretariado dedicado, apoio de enfermagem, psicologia e/ou de outras especialidades médicas em proximidade, permitido a otimização de recursos. Estas consultas temáticas podem ter relações mais estreitas com os centros de referência e alguma atividade formativa e/ou de investigação, embora sem necessidade de afiliação a unidade de ensino e/ou investigação.

\subsection{Centros de Cefaleias ou Unidades de Referência}

Estas unidades são destinadas a casos resistentes, refratários $^{34}$ ou complexos, que se estimam em 1450 por milhão - I4 500 a nível nacional. Considerando um tempo de consulta de 30 a 45 minutos $^{32}$ para estes doentes mais complexos e a necessidade média de 4 consultas anuais, estes doentes necessitarão de 58000 consultas anuais, ou seja, I 200 por semana, a nível nacional. Em termos de tempo, estas representam 29000 a 43500 horas de trabalho especializado anuais, o equivalente a 604 a 836 horas por semana, ou 15 a 21 especialistas de 
neurologia-cefaleias a trabalhar a tempo inteiro, a nível nacional.

Este tipo de unidades altamente diferenciadas só se justificam em centros de nível I, dado que o seu objetivo é serem centros de excelência de cuidados, educação e investigação; devem concentrar a experiência em cefaleias primárias, secundárias e nevralgias raras, serem inovadores e/ou adotantes precoces de novas tecnologias e terapêuticas. ${ }^{8}$ Para atingirem estes objetivos, devem idealmente ser geridos por especialistas com diferenciação em cefaleias - o que, no contexto nacional, pode ser reconhecido pela experiência clínica e/ou diferenciação académica na área - devendo idealmente integrar neurologistas com interesse em cefaleias numa equipa multidisciplinar, que pode incluir psicólogos e/ ou psiquiatras com interesse na gestão da dor crónica, fisioterapeutas e/ou fisiatras com treino específico e enfermagem dedicada, entre outros. Adicionalmente, devem ter acesso a outros especialistas incluindo em neurorradiologia, neurocirurgia, oftalmologia, otorrinolaringologia, ortopedia, reumatologia, pediatria, ginecologia, medicina dentária ou cirurgia maxilofacial, anestesiologia/dor, entre outras. ${ }^{8}$ Nestas unidades deve existir possibilidade de internamento, hospital de dia e acesso a unidade de urgência, com imagiologia e laboratório de apoio permanente. Idealmente, devem estar contidas ou ser afiliadas a uma universidade, hospital escolar ou centro de investigação. ${ }^{8}$

Os profissionais destas unidades deverão:

- ter adquirido as habilitações e competências de forma a serem capazes de efetuar o diagnóstico e gerir todos os doentes com cefaleias primárias e secundárias, de acordo com as orientações diagnósticas e terapêuticas nacionais terapêuticas nacionais $^{24,25}$ e/ou europeias ${ }^{26,27}$;

- dominar de forma autónoma as técnicas interventivas não cirúrgicas de diagnóstico e tratamento de cefaleias primárias e nevralgias;

- manter as suas habilitações através da prática clínica rotineira, dedicando o equivalente a metade do seu tempo de consulta semanal a doentes com cefaleias, em média;

- manter-se atualizado no tema através da participação anual em congressos dedicados e/ou participação em cursos avançados, estudos clínicos e/ou ensaios ou promover investigação sobre o tópico no próprio centro;

- manter a comunicação com os colegas de MGF e neurologistas, quer recebendo, quer retornando os casos que obtenham uma solução estável com um plano delineado e não necessitem manter seguimento em consulta diferenciada.

As funções destas unidades são ${ }^{8}$ :

- providenciar os tratamentos mais diferenciados e indicados para adultos e crianças com cefaleias que não foram geridas com sucesso nos cuidados de saúde primários ou em consultas de neurologia ou consultas monotemáticas, de acordo com os recursos disponíveis;

- dar apoio aos profissionais dos cuidados de saúde primários e neurologistas referenciadores, de forma a orientar e gerir os doentes após intervenção mais diferenciada, quando estáveis;

- providenciar treino clínico específico em cefaleias para qualquer profissional de saúde que se proponha a trabalhar ou a diferenciar-se nesta área;

- contribuir para o desenvolvimento e/ou revisão periódica das orientações terapêuticas nacionais (em colaboração com a Sociedade Portuguesa de Cefaleias e/ou DGS);

- participar na investigação clínica e em ensaios clínicos de valor local, nacional ou internacional;

- participar e/ou promover redes de atividade científica nacionais e/ou internacionais;

- efetuar avaliações periódicas da sua atividade, rentabilidade, sustentabilidade e, idealmente, também da sua qualidade.

Dado que os Centros de nível I têm áreas de referência de até I milhão de habitantes, de forma a garantir que são supridas as necessidades nacionais e há tempo para formação e investigação, cada centro destes deveria ter no mínimo o equivalente a 2 neurologistas em tempo completo exclusivamente para as cefaleias, existindo no máximo 10 destas unidades a nível nacional.

De acordo com a distribuição populacional por zonas, ${ }^{33}$ a região Norte do país tem 3689 713 habitantes (37\%), a região Centro 2327026 (23\%), Lisboa 281585 I (28\%), Alentejo 758739 (8\%) e Algarve 450484 (4\%), pelo que na região Norte deveria existir capacidade para resposta a 5365 utentes - à custa de, no mínimo, o equivalente a 7 a 8 neurologistas a tempo inteiro dedicados às cefaleias, distribuídos por 3 unidades de excelência; do mesmo modo, na região de Lisboa haverá cerca de 4060 utentes, necessitando, no mínimo, do equivalente a 6 neurologistas em tempo inteiro, em 3 unidades; na região Centro 3335 utentes, o equivalente a 5 neurologistas em 2 a 3 centros; no Alentejo e Algarve existem 1740 utentes, necessitando, no mínimo, do equivalente a 2 a 3 neurologistas em um ou dois centros. 


\section{Integração de Cuidados}

\section{I Organigrama}

De acordo com o exposto, propõe-se o seguinte organigrama nacional:

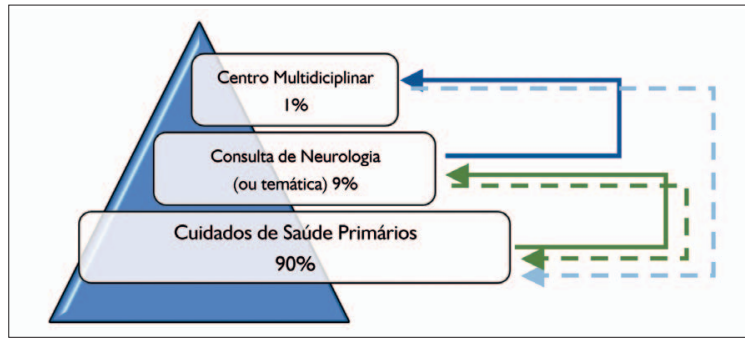

Organigrama. Organização da Prestação de Cuidados se Saúde em Cefaleias - Modelo dos 3 níveis

O reconhecimento e organização de cada tipo de unidade deve ser efetuado pelo serviço de neurologia onde se insere, de acordo com os critérios acima definidos.

O sistema de mobilidade idealmente far-se-á por uma abordagem inicial de proximidade, em MGF, havendo lugar a referenciação para a consulta de Neurologia em casos de difícil controle e, num terceiro tempo, para centros especializados em casos resistentes, refratários, complexos ou com necessidade de recursos inexistentes no local; nos casos em que a situação fique controlada em qualquer dos níveis, os doentes serão remetidos de novo para os cuidados de saúde primários com um plano de seguimento, que pode prever a necessidade de retorno a qualquer um dos níveis, sem obrigatoriedade de re-referenciação ou reinício do processo. Do mesmo modo, se no contexto dos cuidados de saúde primários for identificada situação de elevada complexidade ou com necessidade de terapêutica diferenciada, a referenciação direta para qualquer nível também é possível, assim como a referenciação por outras especialidades médicas, como por exemplo a medicina interna, a reumatologia, a obstetrícia, a pediatria e a oftalmologia, entre outras.

\subsection{Referenciação}

Pressupõe-se, pelo esquema apresentado, que a orientação dos doentes para cada nível de cuidados deverá ser criteriosa, de forma a otimizar a prestação dos mesmos. Assim, propõem-se alguns critérios de referenciação que serão, naturalmente, adaptáveis à realidade de cada unidade.

Referenciação dos cuidados de saúde primários para consultas de neurologia/ monotemáticas ${ }^{35}$ :

- enxaqueca episódica não controlada (definida como com mais que 2 faltas ao trabalho ou idas à urgência por ano e/ou falência de controle das crises após tratamento com 2 preventivos diferentes ou contraindicação para a sua utilização);

- enxaqueca com aura atípica, prolongada, frequente ou com dúvidas de diagnóstico;

- enxaqueca crónica não controlada, com ou sem cefaleia por uso excessivo de medicamentos (definida como manutenção do padrão crónico após falência de tratamento com um preventivo, associada a medidas de controle de ansiedade, sono e excesso de medicação);

- cefaleia tipo tensão crónica ou cefaleia pós-traumática crónica não controladas (definidas por falência de controle da frequência e/ou intensidade da dor após tratamento com 2 preventivos);

- enxaqueca com aura hemiplégica, retiniana ou do tronco cerebral;

- todas as cefaleias trigémino-autonómicas e todas as outras cefaleias primárias;

- nevralgia do trigémio de difícil controle (definida por falência de controle após tratamento com 2 antiepiléticos);

- todas as outras nevralgias cranianas;

- cefaleias desencadeadas por estímulos específicos;

- cefaleias relacionadas com outras patologias neurológicas (do sono, vasculares etc.)

- qualquer cefaleia associada à existência de comorbilidades e/ou situações especiais (ex. gravidez) que limitem a prescrição dos fármacos mais comuns;

- suspeita de cefaleia secundária cujo diagnóstico e/ ou plano terapêutico necessitem de abordagem clínica e/ou recursos não disponíveis nos cuidados de saúde primários;

- incerteza no diagnóstico.

Referenciação das consultas de Neurologia/temáticas para centros de referência:

- enxaqueca episódica resistente ${ }^{34}$ (definida como falência de controle das crises após tratamento com 3 preventivos em dose e duração terapêutica adequada e/ou limitação da utilização destes fármacos e controlo das comorbilidades associadas) e/ ou enxaqueca que condicione mais que 2 faltas ao trabalho ou idas à urgência por ano;

- enxaqueca crónica de difícil controlo, com ou sem cefaleia por uso excessiva de medicamentos com manutenção do padrão crónico após dois preventivos e/ou limitação da utilização destes fármacos, associado a medidas de controlo de ansiedade e sono 
e limitação do uso excessivo de medicamentos;

- enxaqueca crónica com indicação para tratamento com toxina botulínica ou anticorpos monoclonais;

- cefaleia por uso excessivo de medicação com indicação para suspensão em regime de hospital de dia e/ou internamento;

- cefaleia tipo tensão crónica ou cefaleia pós-traumática crónica resistente ${ }^{34}$ (definida por falência de controlo da frequência e/ou intensidade da dor após tratamento com 3 preventivos e/ou limitação da utilização destes fármacos e controle das comorbilidades associadas);

- cefaleias trigémino-autonómicas e outras cefaleias primárias de difícil controlo (falência de controlo das crises após tratamento com I a 2 preventivos em dose e duração terapêutica adequada e/ou limitação da utilização destes fármacos);

- nevralgia do trigémio de difícil controlo (definida por falência de controlo da dor após tratamento com associação de 2 fármacos antiepiléticos e antidepressivos com efeito na dor e/ou limitação da utilização destes fármacos);

- outras nevralgias cranianas com indicação para terapêutica por bloqueios anestésicos de nervos periféricos:

- qualquer doente com cefaleias que beneficie de uma abordagem multidisciplinar para controle sintomático ou que necessite de segunda opinião especializada;

- qualquer doente com cefaleias primárias ou secundárias que necessite de abordagem por especialidades não disponíveis nos centros de nível III/IV.

O sistema de referenciação deve ser agilizado de acordo com os sistemas já em uso, no entanto deve permitir a existência de critério de prioridade para suspeitas de cefaleias secundárias graves e para tratamento de nevralgias e cefaleias trigémino-autonómicas.

\subsection{Educação para a saúde}

As cefaleias são entidades clínicas em que uma adequada estratégia de educação para a saúde pode ter um impacto real na qualidade de vida dos doentes, não passando por medidas de profilaxia, mas pelo ensino do autocuidado e, sobretudo, do maior conhecimento da patologia pelos doentes, familiares e empregadores. Estas medidas podem conduzir à tão necessária destigmatização destas patologias, por reduzir uma relevante parte do impacto intercrítico a estas associado. ${ }^{36,37}$

Sugere-se assim que a enxaqueca e outras cefaleias primárias sejam abordadas no Programa Nacional de
Educação para a Saúde, Literacia e Autocuidados, ${ }^{38}$ sugerindo-se por exemplo incluir informação sobre as mesmas no Repositório de Literacia em Saúde e tomando outras medidas, quer na qualificação dos espaços de atendimento do SNS, quer em campanhas de sensibilização a decorrer no âmbito da medicina do trabalho e medicina escolar.

\section{Conclusão}

Neste documento, a SPC efetuou a transposição e adaptação do modelo básico europeu de organização de serviços de saúde para doentes com cefaleias ${ }^{8-10}$ com o qual pretende alertar as direções das unidades de cuidados de saúde primários e dos serviços de Neurologia sobre as necessidades dos doentes com cefaleias e orientar a sua organização interna, tentando garantir que os cuidados de saúde prestados a estes doentes sejam efetivos, eficientes e tenham o mais elevado nível de qualidade. Não se tratando de normas da Direção Geral de Saúde, a sua aplicação dependerá das condições de cada centro, em particular da eficiente gestão dos recursos humanos, da formação de neurologistas nesta temática e no estabelecimento efetivo e eficiente de vias de comunicação/ referenciação entre unidades.

\section{Agradecimentos}

Aos Dr. Filipe Palavra, Dra. Isabel Luzeiro e à Profa. Isabel Pavão Martins, membros da Comissão Científica da Sociedade Portuguesa de Cefaleias, pela revisão crítica do documento e sugestões efetuadas.

\section{Responsabilidades Éticas}

Conflitos de Interesse: Os autores declaram não possuir conflitos de interesse.

Suporte Financeiro: O presente trabalho não foi suportado por nenhum subsidio o bolsa ou bolsa.

Proveniência e Revisão por Pares: Não comissionado; revisão externa por pares.

Ethical Disclosures

Conflicts of Interest: The authors have no conflicts of interest to declare.

Financial Support: This work has not received any contribution grant or scholarship.

Provenance and Peer Review: Not commissioned; externally peer reviewed.

\section{References / Referências}

1. Headache Classification Committee of the International Headache Society (IHS) The International Classification of Headache Disorders, 3rd edition. Cephalalgia. 2018;38:1211. doi: $10.1177 / 0333102417738202$

2. Steiner TJ, Stovner LJ, Vos T, Jensen R, Katsarava Z. Mi- 
graine is first cause of disability in under 50s: will health politicians now take notice? J Headache Pain. 2018;19:17-20.

3. Olesen J, Gustavsson A, Svensson M, Wittchen HU, Jönsson B. The economic cost of brain disorders in Europe. Eur J Neurol. 2012;19:155-62. doi: 10.1111/j.14681331.2011.03590.x

4. Linde M, Gustavsson A, Stovner LJ, Steiner TJ, Barré J, Katsarava $Z$, et al. The cost of headache disorders in Europe: The Eurolight project. Eur J Neurol. 2012;19:703-11.

5. Gustavsson A, Svensson M, Jacobi F, Allgulander C, Alonso $J$, Beghi E, et al. Cost of disorders of the brain in Europe 2010. Eur Neuropsychopharmacol. 2011;21:718-79.

6. World Health Organization. Atlas of headache disorders and resources in the world 2011. Geneva: WHO; 2011.

7. Lipton RB, Scher Al, Kolodner K, Liberman J, Steiner TJ, Stewart WF. Migraine in the United States: Epidemiology and patterns of health care use. Neurology. 2002; 58:88594. doi: $10.1212 / \mathrm{wnl} .58 .6 .885$.

8. Steiner TJ, Göbel H, Jensen R, Lampl C, Paemeleire K, Linde $M$, et al. Headache service quality: The role of specialized headache centres within structured headache services, and suggested standards and criteria as centres of excellence. J Headache Pain. 2019;20:24. doi: 10.1186/ s10194-019-0970-7.

9. Steiner TJ, Antonaci F, Jensen R, Lainez MJA, Lanteri-Minet $M$, Valade $D$. Recommendations for headache service organisation and delivery in Europe. J Headache Pain. 2011:12:419-26

10. Antonaci F, Valade D, Lanteri-Minet M, Láinez JM, Jensen $\mathrm{R}$, Steiner TJ. Proposals for the organisation of headache services in Europe. Intern Emerg Med. 2008;3:25-8.

11. Students M, Sandor P, Lainez M, Purdy A, Schoonman G, Rapoport A. The International Headache Society's Core Curriculum on Headache for Neurologists. $\mathrm{Br} \mathrm{J}$ Pain. 2012;6:103-5.

12. European Headache Federation. EHF Mission. [consultado 16.07.2020] Disponível em https://ehf-org.org/ehf-mission/

13. Pereira-Monteiro J. Cefaleias: Estudo epidemiológico e clínico numa população urbana. Porto; Sociedade Portuguesa de Neurologia;1995.

14. Instituto Nacional de Estatística. CENSOS 2011, Portugal. [consultado 16.07.2021] Disponível em https://censos.ine.pt/

15. SNS. Redes de Referenciação Hospitalar.

16. Barros J, Silveira L, Tavares F, Oliveira F, Natário A, Cunha L. Rede de Referenciação Hospitalar de Neurologia. [consultado Jan 2020] Disponível em https://www.sns.gov. pt/wp-content/uploads/2018/03/RRH-Neurologia-aprovada-26-03-2018.pdf

17. Ministério da Saúde Português. Portaria n.o 195/2016. Diário da República no 137/2016, Série | 2016-07-19 [Internet]. 2016;2311-2. [consultado Jan 2020] Disponível em https://data.dre.pt/eli/port/195/2016/07/19/p/dre/pt/html

18. Programa Nacional de Educação para a Saúde,Literacia e Autocuidados [consultado Jan 2020] Disponível em https:// www.dgs.pt/em-destaque/programa-nacional-de-educacao-para-a-saude-literacia-e-autocuidados.aspx\%0D\%0A

19. Sociedade Portuguesa de Cefaleias.[consultado Jan 2020] Disponível em http://www.cefaleias-spc.com/

20. Conselho Nacional do Internato Médico. Programa formativo da Formação Especializada de Medicina Geral e Familiar. In: Republica Portuguesa ., editor. Portaria no 125/2019 Diário da República no 83/2019, Série I de 2019-04-302019. 2019.

21. Latinovic R, Gulliford M, Ridsdale L. Headache and migraine in primary care: Consultation, prescription, and referral rates in a large population. J Neurol Neurosurg Psychiatry. 2006; 77:385-7. doi: 10.1136/jnnp.2005.073221.

22. James SL, Abate $D$, Abate KH, Abay SM, Abbafati C, Abbasi $\mathrm{N}$, et al. Global, regional, and national incidence, prevalence, and years lived with disability for 354 Diseases and Injuries for 195 countries and territories, 1990-2017: A systematic analysis for the Global Burden of Disease Study 2017. Lancet. 2018; 392:1789-858. doi: 10.1016/S01406736(18)32279-7

23. Gil-Gouveia R. Headache from the doctors' perspective. Eur Neurol. 2014;71:157-64.

24. Monteiro JMP, Ribeiro CAF, Luzeiro IMDS, Machado MGDASF, Esperança PM. Recomendações terapêuticas para cefaleias: I parte. Cefaleias primárias - Edição conjunta da: Sociedade Portuguesa de Neurologia e da Sociedade Portuguesa de Cefaleias. Sinapse. 2009 9(2) Suppl 1: 1-36.

25. Sociedade Portuguesa de Cefaleias. Recomendações terapêuticas para cefaleia primárias. Sinapse. 2021. 21:1-100.

26. Evers S, Áfra J, Frese A, Goadsby PJ, Linde M, May A, et al. EFNS guideline on the drug treatment of migraine - Revised report of an EFNS task force. Eur J Neurol. 2009;16:968-81.

27. Sacco S, Bendtsen L, Ashina M, Reuter U, Terwindt G, Mitsikostas DD, et al. European headache federation guideline on the use of monoclonal antibodies acting on the calcitonin gene related peptide or its receptor for migraine prevention. J Headache Pain. 2019; 20:6. doi: 10.1186/s10194018-0955-y.

28. Botelho H. Constituição de Listas de Utentes por Médico de Família - Recomendação $n^{\circ}$ 2.2017. [consultado 16.7.21] Disponível em https://www.sns.gov.pt/wp-content/uploads/2018/08/cncsp_recomendacao_2017_02.pdf

29. Secretaria de Estado da Saúde. Programa de formação da especialidade de Neurologia. Portaria n.o 376/2012. Diário da República n.o 223/2012, Série I de 2012-11-192012. 2012.

30. Danish Headache Center. Master of Headache Disorders, 2020. [consultado 16.7.21] Disponível em https://mhd.ku.dk/

31. University of Rome. Master Degree in Headache Medicine. 2021. [consultado 16.7.21] Disponível em https://web. uniroma1.it/masterheadache/

32. República D. Regulamento dos tempos padrão das consultas médicas [Internet]. Vol. 178, Diário da República Regulamento n.o 724/2019. 2019. p. 103-18.: [consultado Jan 2020]Disponível em:https://dre.pt/home/-/dre/124750410/ details/3/maximized?serie $=\| \&$ dreld $=124721053$

33. Entidade Reguladora da Saúde. Entidade Reguladora da Saúde - Estudo para a Carta Hospitalar. 2012 [consultado Jan 2020] Disponível em: https://www.ers.pt/uploads/writer_file/document/668/ERS_Carta_Hospitalar.vf.pdf

34. Sacco $S$, Braschinsky M, Ducros A, Lampl C, Little $P$, van den Brink $A M$, et al. European headache federation consensus on the definition of resistant and refractory migraine : Developed with the endorsement of the European Migraine \& Headache Alliance (EMHA). J Headache Pain. 2020; 21:76. doi: 10.1186/s10194-020-01130-5.

35. Steiner TJ, Jensen R, Katsarava Z, Linde M, MacGregor EA, Osipova $\mathrm{V}$, et al. Aids to management of headache disorders in primary care (2nd edition) : on behalf of the European Headache Federation and Lifting The Burden: the Global Campaign against Headache. J Headache Pain. 2019;20:57. doi: 10.1186/s10194-018-0899-2

36. Gallardo V, Trochet J, Torres-Ferrus M, Sanchez del Rio M, Lainez J, Leira R et al. eMIG (I): the impact of digital platforms focused on migraine patient-centered outcome research. London: Migraine Trust International Symposium; London; 2018.

37. Buse DC, Scher Al, Dodick DW, Reed ML, Fanning KM, Manack Adams A, et al. Impact of Migraine on the Family: Perspectives of People with Migraine and Their Spouse/Domestic Partner in the CaMEO Study. Mayo Clin Proc. 2016;S0025-6196:00126-9. doi: 10.1016/j.mayocp.2016.02.013

38. Diário da República . Programa Nacional de Educação para a Saúde, Literacia e Autocuidados. 2017. 